\title{
Phylogenetic Relationship of the Twenty-One DNA Groups of the Genus Acinetobacter as Revealed by 16S Ribosomal DNA Sequence Analysis
}

\author{
ASHRAF IBRAHIM,${ }^{1}+$ PETER GERNER-SMIDT,${ }^{1 *}$ AND WERNER LIESACK ${ }^{2}$ \\ Department of Clinical Microbiology, Statens Seruminstitut, Copenhagen, DK-2300, Denmark, ${ }^{1}$ and Max-Planck- \\ Institut für Terrestrische Mikrobiologie, D-35043 Marburg, Germany ${ }^{2}$
}

\begin{abstract}
The inter- and intrageneric relationships of members of the genus Acinetobacter were investigated by performing a comparative sequence analysis of PCR-amplified 16S ribosomal DNAs (rDNAs) from 21 strains representing all of the DNA groups that have been described. Phylogenetic treeing confirmed that Acinetobacter spp. form a coherent cluster within the gamma subdivision of the class Proteobacteria that includes strains with overall levels of $16 \mathrm{~S}$ rDNA sequence similarity of more than $94 \%$. The analysis of intrageneric relationships suggested that the majority of the strains cluster in five clearly distinguishable clusters, and this conclusion was supported by the results obtained with the different methods used for phylogenetic analysis (i.e., the maximum-likelihood, parsimony, and distance matrix methods). The first cluster contains the representatives of DNA groups 2 (Acinetobacter baumannii) and TU13, whereas the second cluster comprises representatives of DNA groups 3, "Close To TU13," and "between 1 and 3." The representatives of closely related Acinetobacter DNA groups 8 (Acinetobacter lwoffii) and 9 belong to the third cluster, which includes the representative of DNA group 6 as well. The fourth cluster is formed by DNA groups BJ15, BJ16, and BJ17, and the fifth cluster comprises DNA groups 1 (Acinetobacter calcoaceticus), BJ14, 10, and 11. Within the fifth cluster the 16S rDNA sequences of DNA group 10 and 11 strains are nearly identical. The representatives of DNA groups 4 (Acinetobacter haemolyticus), 5 (Acinetobacter junii), 7 (Acinetobacter johnsonii), 12 (Acinetobacter radioresistens), TU14, and TU15 form individual branches that are not significantly affiliated with any of the five clusters identified. Apart from the clustering of the most closely related DNA groups, the general topology of the distance dendrogram revealed some discrepancy with previous DNA-DNA hybridization data, which may point to the inadequacy of comparative 16S rDNA sequence analysis for reflecting true evolutionary relationships of closely related bacterial taxa. Important, however, was the presence of unique sequence motifs in each of the 21 different DNA groups studied, which may be useful for rapid differentiation of DNA groups of the genus Acinetobacter.
\end{abstract}

Members of the genus Acinetobacter are ubiquitously distributed in nature. They are gram-negative, strictly aerobic, nonmotile, diplococcoid rods that are oxidase negative and catalase positive. Taxonomically, the genus Acinetobacter has a rather long and complicated history. Although at least 21 different DNA groups (genomospecies) have been reported in the literature, only seven species have been delineated. The most recent classification of members of the genus Acinetobacter is the classification of Bouvet and Grimont (1). A total of 85 strains were characterized by DNA-DNA hybridization, and 74 strains were placed in 12 different DNA groups, each comprising strains that exhibited more than $70 \%$ DNA-DNA relatedness and $\Delta T_{m}$ values of $5^{\circ} \mathrm{C}$ or less $\left(\Delta T_{m}\right.$ is the difference between the melting temperature of a homologous hybrid and the melting temperature of a heterologous hybrid). Based on this study, the following four new species were named: Acinetobacter baumannii, Acinetobacter junii, Acinetobacter johnsonii, and Acinetobacter haemolyticus. In addition, the descriptions of the old species Acinetobacter calcoaceticus and Acinetobacter lwoffii were emended. In 1988, an additional species, Acinetobacter radioresistens, was described (17). Tjernberg and Ursing showed by using DNA-DNA hybridization that this

\footnotetext{
* Corresponding author. Mailing address: Department of Gastrointestinal Infection, Statens Seruminstitut, Copenhagen, DK-2300 Denmark. Phone: 45 32683268. Fax: 45 32683873. E-mail: PGS@SSI.DK.

$\dagger$ Present address: Department of Microbiology, National Defence Research Institute, Umeå S-901-82, Sweden.
}

species corresponded to DNA group 12 defined by Bouvet and Grimont (21). Moreover, Tjernberg and Ursing verified the existence of all of the DNA groups described by Bouvet and Grimont but were unable to discriminate between the closely related DNA groups 8 and 9. Also, three additional DNA groups were described and subsequently designated DNA groups 13 through 15 . Concurrently, Bouvet and Jeanjean delineated five new proteolytic DNA groups and designated them DNA groups 13 through 17 (2). DNA group 14 of Tjernberg and Ursing corresponds to DNA group 13 of Bouvet and Jeanjean. Recently, two other new DNA groups were described (11). One group was found to be closely related to DNA group 13 of Tjernberg and Ursing. The other group was found to have DNA-DNA hybridization values between those of DNA groups 1 and 3. On the one hand, in every DNA hybridization study on Acinetobacter spp. done so far, several strains have been left ungrouped, suggesting that the genus is rather diverse genotypically. On the other hand, the 21 DNA groups cannot be differentiated on the basis of the currently available phenotypic traits. In spite of our knowledge about the genotypic diversity within the genus Acinetobacter and overlaps in the results of the DNA-DNA hybridization studies that have been performed, a comprehensive $16 \mathrm{~S}$ ribosomal DNA (rDNA)-based analysis to complement the existing DNA-DNA hybridization data has not been performed previously. Consequently, it was the objective of this study to sequence PCRamplified 16S rDNAs of representatives of all of the Acinetobacter DNA groups presently known to elucidate the 
TABLE 1. Acinetobacter DNA groups investigated and 16S rDNA accession numbers

\begin{tabular}{|c|c|c|c|}
\hline Strain & DNA group & Species & $\begin{array}{c}\text { Accession } \\
\text { no. }\end{array}$ \\
\hline $\operatorname{ATCC} 23055^{\mathrm{T}}$ & 1 & A. calcoaceticus & Z93434 \\
\hline ATCC $19606^{\mathrm{T}}$ & 2 & A. baumannii & Z93435 \\
\hline ATCC 17922 & 3 & Unnamed & Z93436 \\
\hline ATCC $17906^{\mathrm{T}}$ & 4 & A. haemolyticus & Z93437 \\
\hline ATCC $17908^{\mathrm{T}}$ & 5 & A. junii & Z93438 \\
\hline АТCC 17979 & 6 & Unnamed & Z93439 \\
\hline ATCC $17909^{\mathrm{T}}$ & 7 & A. johnsonii & Z93440 \\
\hline ATCC 17925 & 8 & A. lwoffii & Z93441 \\
\hline ATCC 9957 & 9 & Unnamed & Z93442 \\
\hline ATCC 17924 & 10 & Unnamed & Z93443 \\
\hline ATCC 11171 & 11 & Unnamed & Z93444 \\
\hline (M) $17694^{\mathrm{T}}$ & 12 & A. radioresistens & Z93445 \\
\hline ATCC 17903 & TU13 & Unnamed & Z93446 \\
\hline ATCC 17905 & TU14 (BJ13) & Unnamed & Z93447 \\
\hline (M) $151 \mathrm{a}$ & TU15 & Unnamed & Z93448 \\
\hline 10090 & CTTU13 & Unnamed & Z93449 \\
\hline 10095 & $1-3$ & Unnamed & Z93450 \\
\hline 382 & BJ14 & Unnamed & Z93453 \\
\hline 79 & BJ15 & Unnamed & Z93452 \\
\hline 78 & BJ16 & Unnamed & Z93451 \\
\hline 942 & $\mathrm{BJ} 17$ & Unnamed & Z93454 \\
\hline
\end{tabular}

meaningfulness of comparative $16 \mathrm{~S}$ rDNA sequence analysis and to evaluate the diagnostic potential of highly variable regions for reliable differentiation of the DNA groups based on characteristic sequence motifs.

\section{MATERIALS AND METHODS}

Bacterial strains. A total of 21 bacterial isolates were analyzed (Table 1). The type strains of six of the seven validly described Acinetobacter species were included, as were representatives of all of the other DNA groups described by different investigators. The type strain of the seventh described species, $A$. lwoffii (DNA group 8), was not included in the study because of uncertainty about its taxonomic position (21). Instead, two other reference strains (ATCC 17925 and ATCC 9957) were investigated as representatives of DNA groups 8 and 9 described by Bouvet and Grimont (1). This was done to be sure to include strain belonging to both of these DNA groups in the study. The strains were obtained from the American Type Culture Collection and our own culture collection (strains 10090 and 10095) or were kindly donated by Philippe Bouvet, Institut Pasteur (strains 382, 79, 78, and 942) and Ingela Tjernberg, Malmö, Sweden [strains (M) $17694^{\mathrm{T}}$ and (M) 151a]. For the DNA groups for which two different numbering systems exist, the DNA group numbers preceded by the initials of the authors that described them are used (i.e., Tjernberg-Ursing DNA groups TU13 through TU15 [21] and Bouvet-Jeanjean DNA groups BJ13 through BJ17 [2]). DNA groups "Close To TU13" and "between 1 and 3" (11) are designated "CTTU13" and "1-3," respectively.

Growth conditions and DNA preparation. Test bacteria were grown overnight on 5\% defibrinated horse blood agar (catalog no. 677) plates prepared by workers in the Substrate Department of the Statens Seruminstitut. Single colonies were picked up and washed in $1 \mathrm{ml}$ of TE buffer, pelleted, and resuspended in $100 \mu \mathrm{l}$ of distilled water. Cells were lysed and DNA was extracted as described by Ibrahim et al. (12).

PCR amplification and sequencing of 16S rDNA. For amplification of the $16 \mathrm{~S}$ rDNA, the following reagents were combined in a final volume of $50 \mu \mathrm{l}$ : each deoxynucleoside triphosphate at a concentration of $100 \mu \mathrm{M}, 10$ pmol of each primer, $1 \times$ Taq buffer (Boehringer, Mannheim, Germany), and approximately $10 \mathrm{ng}$ of purified RNase-treated template DNA. After $50 \mu \mathrm{l}$ of mineral oil was added to each tube, the tubes were heated to $80^{\circ} \mathrm{C}$, and $1 \mathrm{U}$ of $\mathrm{Taq}$ polymerase (Boehringer) was subsequently added. Amplification was carried out with a model 480 DNA thermal cycler (Perkin-Elmer, Allerød, Denmark) programmed for 30 cycles, with each cycle consisting of $1 \mathrm{~min}$ at $94^{\circ} \mathrm{C}$ (denaturation), $1 \mathrm{~min}$ at $52^{\circ} \mathrm{C}$ (annealing), and $1 \mathrm{~min}$ at $72^{\circ} \mathrm{C}$ (extension). A final extension step at $72^{\circ} \mathrm{C}$ was performed for $3 \mathrm{~min}$. The $5^{\prime}$ and $3^{\prime}$ primers used were $5^{\prime}$ GAGTTTGATC CTGGCTCAG3' and 5'ACGGCTACCTTGTTACGACTT3', targeting conserved regions 9 to 27 and 1512 to 1492 (IUB nomenclature for Escherichia coli [3]). The $16 \mathrm{~S}$ rDNA products were purified by separation on $1.5 \%$ low-melting point agarose gels. Gel fragments containing the desired product were sliced and digested with Gelase enzyme (Epicentre Technologies, Madison, Wis.) as described elsewhere (12). Sequencing was carried out nonradioactively by using a Taq DyeDeoxy terminator cycle sequencing kit (Applied Biosystems, Foster City, Calif.) according to the manufacturer's instructions. The sequence primers used were those described by Lane (14). Sequence products were analyzed with a model 373A DNA sequencer (Applied Biosystems).

Data analysis. The $2116 \mathrm{~S}$ rDNA sequences of the Acinetobacter spp. studied were manually aligned and compared with reference sequences of members of the gamma subdivision of the class Proteobacteria by focusing on members of the most closely related genera. The reference sequences were obtained from public databases (EMBL [5] and Ribosomal Database Project [15]). The intrageneric relationships of the Acinetobacter spp. shown in Fig. 1 were deduced by comparing only the positions of a continuous stretch of 1,389 nucleotides corresponding to positions 28 through 1438 of the $16 \mathrm{~S}$ rRNA of $E$. coli (International Union for Biochemistry nomenclature) from which unambiguous sequence information could be obtained. Three different methods of phylogenetic analysis supplied by the PHYLIP package, version 3.5c (8), were used to delineate intrageneric relationships. These methods were the maximum-likelihood method (dnaml program), the parsimony method (dnapars program), and the neighbor-joining method with a distance matrix calculated with the Jukes-Cantor correction (13)

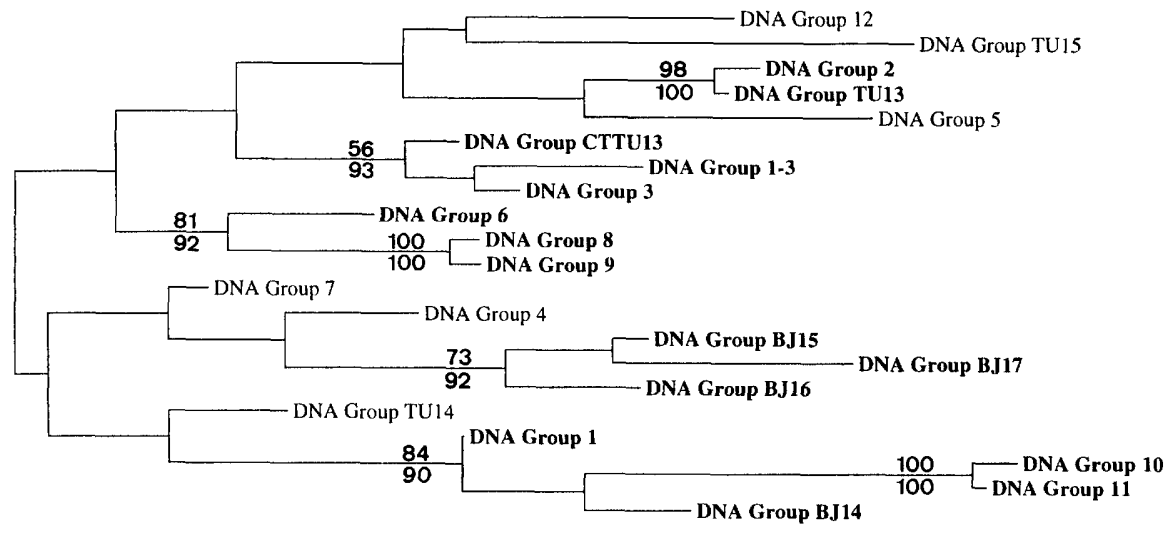

FIG. 1. Unrooted phylogenetic dendrogram constructed for 21 strains representing all known DNA groups described for the genus Acinetobacter. The tree was constructed by performing a maximum-likelihood analysis. The percentages of 100 bootstrap resamplings that supported the presence of five distinct clusters in the parsimony analysis (above the lines) and in the neighbor-joining analysis (below the lines) are indicated. The thresholds for defining these clusters were bootstrap values of at least $50 \%$ in the parsimony analysis and, for the same nodes simultaneously, at least $90 \%$ in the neighbor-joining analysis. Scale bar $=0.02$ estimated base change per nucleotide. 


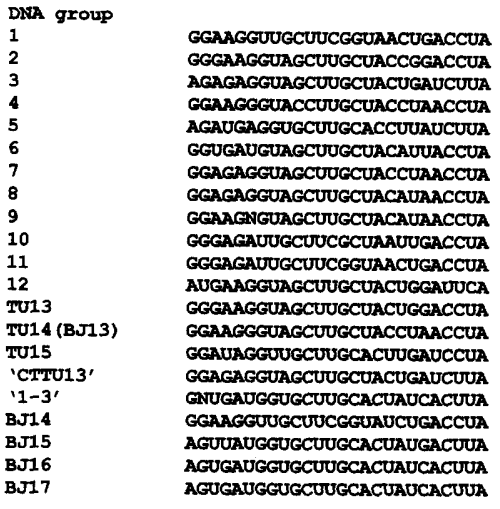

FIG. 2. Characteristic sequence motifs for the 21 Acinetobacter DNA groups at the helix 6 variable region $(22,23)$, corresponding to $E$. coli $16 \mathrm{~S}$ rRNA positions 70 to 101 (IUB nomenclature for $E$. coli). The stretch shown has a deletion of six nucleotides compared to the $E$. coli sequence.

The bootstrap method (7) (PHYLIP package) was used to check the topology pertubations in the neighbor-joining and parsimony trees.

Nucleotide sequence accession numbers. The nucleotide sequences used in this study have been deposited in the EMBL Nucleotide Sequence Database under accession no. Z93434 through Z93454 (Table 1).

\section{RESULTS AND DISCUSSION}

The PCR primer pair used resulted in amplification of almost complete 16S rRNA genes from the 21 strains studied. Phylogenetic treeing performed with a restricted number of nucleotide positions confirmed previous reports that members of the genus Acinetobacter form a phylogenetically coherent group of organisms within the gamma subdivision of the class Proteobacteria and that species of the genus Moraxella represent the most closely related lineage (data not shown) $(6,18)$. In order to elucidate the intrageneric relationships of the known DNA hybridization groups of the genus Acinetobacter, almost complete $16 \mathrm{~S}$ rRNA genes were comparatively analyzed. The overall levels of $16 \mathrm{~S}$ rDNA sequence similarity for the strains were greater than $94 \%$. The analysis of intrageneric relationships in which the maximum-likelihood method was used revealed that the majority of the strains can be assigned to five different lineages (Fig. 1). Almost identical tree topologies were obtained when the parsimony method or the neighbor-joining method based on a Jukes-Cantor distance matrix was used to construct the tree. Bootstrap analyses in which the confidence levels of the interior branches of the parsimony and neighbor-joining trees were tested resulted in characterization of five clearly distinguishable clusters; however, the degrees of statistical significance differed. When threshold values of more than $50 \%$ were used for nodes in the parsimony tree and threshold values of more than $90 \%$ were used for nodes in the neighbor-joining tree, the five clusters were defined as follows: cluster I, including DNA groups 2 and TU13; cluster II, including DNA groups 3 , CTTU13, and 1-3; cluster III, including DNA groups 6, 8, and 9 (with DNA groups 8 and 9 having nearly identical sequences); cluster IV, including DNA groups BJ15 through BJ17; and cluster $\mathrm{V}$, including DNA groups 1, $\mathrm{BJ} 14,10$, and 11 . Within cluster $\mathrm{V}$ the representatives of DNA groups 10 and 11 have almost identical $16 \mathrm{~S}$ rDNA sequences. The representatives of DNA groups $4,5,7,12$, TU14, and TU15 form individual branches that are not significantly affiliated with any of the five clusters identified (based on the bootstrap threshold values defined above). The outcome of the treeing analysis was the same whether an outgroup sequence

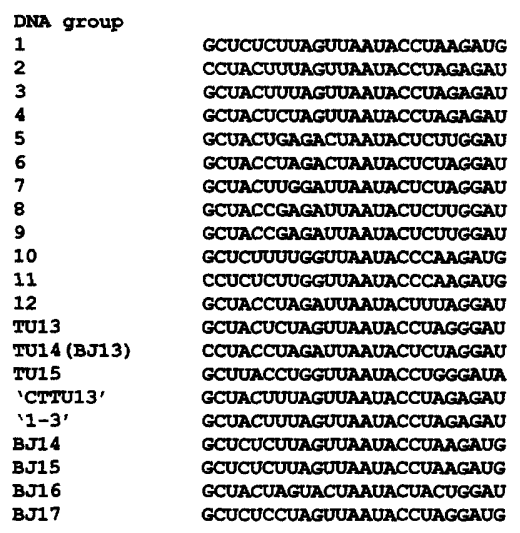

FIG. 3. Characteristic sequence motifs for Acinetobacter DNA groups at the helix 18 variable region $(22,23)$ compared to the $E$. coli $16 \mathrm{~S}$ rRNA sequence at positions 453 to 477 (IUB nomenclature for $E$. coli).

(the 16S rDNA sequence of Pseudomonas aeruginosa [accession no. M34133]) was used to root the tree or not. Our data are consistent with the results of a previous study in which the authors used 16S rDNA sequences of representatives of the seven named Acinetobacter species and DNA group 11 (18).

A search for nucleotide positions at which the nucleotides are the same for all of the strains belonging to one of the five defined clusters but are not found in any other strain or are found in only a few other strains resulted in a set of signatures which support to some degree the phylogenetic placement of the majority of the Acinetobacter spp. into the five different subgroups (Fig. 1 and Table 2). The representatives of cluster I (DNA groups 2 and TU13) have a guanosine at position 648 (IUB nomenclature for $E$. coli), which is unique for these two

TABLE 2. Sequence motifs that support the presence of the five distinct groups derived from the phylogenetic analysis ${ }^{a}$

\begin{tabular}{|c|c|c|c|c|c|}
\hline \multirow[b]{2}{*}{ Position $^{b}$} & \multicolumn{5}{|c|}{ Nucleotide in: } \\
\hline & $\begin{array}{c}\text { Cluster } \\
I^{c}\end{array}$ & $\begin{array}{l}\text { Cluster } \\
\text { II }\end{array}$ & $\begin{array}{c}\text { Cluster } \\
\text { III }\end{array}$ & $\begin{array}{l}\text { Cluster } \\
\text { IV }\end{array}$ & $\begin{array}{c}\text { Cluster } \\
\mathrm{V}\end{array}$ \\
\hline 79 & G & $\mathrm{G}$ & G & $\mathrm{G}$ & $\mathbf{T}^{* d}$ \\
\hline 81 & A & $\mathrm{A} / \mathrm{T}$ & A & $\mathrm{T}$ & $\mathbf{G}^{*}$ \\
\hline 82 & G & G & $G$ & $\mathrm{G}$ & $C^{*}$ \\
\hline 83 & $\mathrm{C}$ & $\mathrm{C}$ & C & $\mathrm{C}$ & $\mathrm{T}^{*}$ \\
\hline 85 & $\mathrm{~T}$ & $\mathrm{~T}$ & $\mathrm{~T}$ & $\mathrm{~T}$ & $C^{*}$ \\
\hline 206 & $\mathrm{~T}$ & $\mathrm{~T}$ & C & $\mathrm{T}$ & $\mathrm{T}$ \\
\hline 213 & A & A & $\mathbf{G}$ & A & A \\
\hline 586 & $\mathrm{C}$ & $\mathrm{C}$ & $\mathbf{T}$ & $\mathrm{C}$ & $\mathrm{C}$ \\
\hline 591 & $\mathrm{~T}$ & A & A & G & $\mathrm{T}$ \\
\hline 614 & $\mathrm{C}$ & $\mathrm{C}$ & $\mathrm{C}$ & $\mathbf{T}$ & C \\
\hline 626 & $\mathrm{G}$ & G & $G$ & $\mathbf{A}$ & G \\
\hline 648 & G* & $\mathrm{T}$ & $\mathrm{T}$ & C & A \\
\hline 658 & A & $\mathbf{G}^{*}$ & A & A & A \\
\hline 755 & G & G & $\mathbf{A}$ & $\mathrm{G}$ & G \\
\hline
\end{tabular}

${ }^{a}$ See Fig. 1.

${ }^{b}$ Positions according to the IUB nomenclature for $E$. coli.

${ }^{c}$ Clusters are defined as shown in Fig. 1. Cluster I contains DNA groups 2 and TU13; cluster II contains DNA groups CTTU13, 1-3, and 3; cluster III contain DNA groups 6, 8, and 9; cluster IV contains DNA groups BJ15 through BJ17; and cluster V contains DNA groups 1, BJ14, 10, and 11 .

${ }^{d}$ The nucleotides which are highly indicative for clusters are in boldface type. These nucleotides are either unique for one of the defined subgroups (indicated by an asterisk) or are found in a very few of the other Acinetobacter spp. outside the five clusters. 
sequences in the 21 sequences of Acinetobacter strains studied. Similarly, the representatives of cluster II (DNA groups 3, CTTU13, and 1-3) are characterized by a unique guanosine at position 658 ( $E$. coli numbering). The representatives of cluster III (DNA groups 6, 8, and 9) are defined by two coordinated nucleotide substitutions. The first of these is a C-G base pair at positions 206 and 213 (E. coli numbering), which is shared only by the representative of DNA group 12 . The second coordinated substitution is a T-A base pair at positions 586 and 755 (E. coli numbering) that is shared only by the representative of DNA group TU15. The members of cluster IV (DNA groups $\mathrm{BJ} 15$ through $\mathrm{BJ} 17$ ) are also characterized by two coordinated nucleotide substitutions. The first coordinated substitution is a G-C base pair at positions 591 and 648 (E. coli numbering), which is shared only by the representative of DNA group 4, and the second coordinated substitution is a T-A base pair at positions 614 and 626 (E. coli numbering), which is shared by the representatives of DNA groups 4 and 7 . The finding that in addition to members of cluster IV, both of these coordinated nucleotide substitutions are also present in the representative of DNA group 4 suggests that the DNA group 4 strain may be a member of cluster IV, which is also consistent with the topology of the tree shown in Fig. 1. Five unique nucleotides are highly indicative for members of cluster V (DNA groups 1, 10,11 , and BJ14). These nucleotide signatures, which are not found in any of the remaining 17 strains, are located in a highly variable region characterized by helix 6 (numbering of Van de Peer et al. [22, 23]) and include the nucleotides at positions 79 $(\mathrm{T}), 81(\mathrm{G}), 82(\mathrm{C}), 83(\mathrm{~T})$, and $85(\mathrm{C})$ (E. coli numbering).

We did not include the type strain of $A$. lwoffii in this study because of the uncertainty of its taxonomic affiliation. Bouvet and Grimont (1) placed the type strain of $A$. lwoffii (NCTC 5866) and strain ATCC 17925 in DNA group 8 and strain ATCC 9957 in DNA group 9. In the study of Tjernberg and Ursing (21), type strain NCTC 5866 and strain ATCC 9957 clustered together, whereas strain ATCC 17925 clustered at a marginal position in their DNA group $8 / 9$ ( $A$. lwoffii). In order to examine strains belonging to both DNA group 8 and DNA group 9 described by Bouvet and Grimont (1), strains ATCC 9957 and ATCC 17925 were selected for the present study. These two strains had almost identical rDNA sequences.

When our data are compared with previously published DNA-DNA hybridization data $(1,2,11,21)$, both similarities and differences are found. As determined by DNA-DNA hybridization, DNA groups 1 through 3, TU13, CTTU13, and 1-3 form a tight cluster (the $A$. calcoaceticus- $A$. baumannii complex) (10); proteolytic DNA groups 4 through 6, TU14, and $\mathrm{BJ} 14$ through $\mathrm{BJ} 17$ are also more closely related to each other than to other acinetobacters $(1,2,21)$, as are DNA groups 7 through 9 and TU15 $(1,21)$; DNA groups 10 and 11 are related as determined by DNA hybridization; and DNA group 12 is a separate entity $(1,21)$. rDNA sequencing confirmed the close relationship between DNA groups 10 and 11 and the close relationship between DNA groups 8 and 9 . The unique position of DNA group 12 within the genus determined in all previous studies was also reproduced. However, the DNA groups in the $A$. calcoaceticus- $A$. baumannii complex were split into three clusters (clusters II, III, and V). The very close relationship between DNA groups 2 and TU13 was confirmed, but DNA group CTTU13 clustered with DNA groups 3 and 1-3. In cluster $\mathrm{V}$, DNA group 1 was placed with DNA groups 10,11 , and BJ14. Likewise, the proteolytic DNA groups were distributed in different clusters, with only the rDNA sequences of DNA groups BJ15 through BJ17 identified as a tight cluster. Also, DNA groups TU15 and 7 are separated from each other and from the strains of DNA group $8 / 9$ by the rDNA sequence analysis data. One important reason for the discrepancies between the results of the $16 \mathrm{~S}$ rDNA analysis and the DNA-DNA hybridization data may be the fact that the majority of the nucleotide differences between the strains that were investigated occur in highly variable regions as defined by Van de Peer et al. (22). Nucleotide positions in these areas are known to have a very fast evolutionary rate, based on fixation of random mutation events resulting in a loss of phylogenetic information. In addition, lateral gene transfer between closely related bacteria followed by gene conversion leading to homogenization of multiple rRNA gene copies in the genomes of individual lineages may eclipse fast mutational rates in the highly variable regions (4). However, the meaningfulness of the highly variable regions for phylogenetic inference is still under investigation (20) and may vary with the bacterial group being studied. Therefore, sequence motifs, especially those like coordinated substitutions between distantly located sequence positions as determined for members of cluster III (T-A base pair at positions 586 and 755) and members of cluster IV (G-C pair at positions 591 and 648), represent helpful markers for inferring true phylogenetic affiliations among closely related microorganisms, like the Acinetobacter spp.

In addition, the levels of DNA-DNA relatedness in the proteolytic groups vary between 33 and $68 \%$, with DNA group 5 being least similar to the other groups. Thus, the DNA-DNA clustering of these proteolytic groups may not be reliable, and it may not be surprising that they are split into different $16 \mathrm{~S}$ rDNA clusters. Consequently, in the present situation, reliable correlation between the 16S rDNA sequence analysis and DNA-DNA hybridization is difficult to achieve.

At variance with the $16 \mathrm{~S}$ rDNA sequence data but consistent with the DNA-DNA hybridization data is the fact that the gyrB gene sequence analysis data reported by Yamamoto and Harayama (24) revealed a close relationship among strains in the $A$. calcoaceticus- $A$. baumannii complex. However, only strains belonging to DNA groups 1 through 3 in this complex were investigated in the study of Yamamoto and Harayama. Strains belonging to the proteolytic DNA groups were also found to be more closely related to each other on the basis of gyr $B$ gene data than on the basis of $16 \mathrm{~S}$ rDNA sequence analysis data. Yet, protein-encoding genes like gyrB evolve much faster than rRNA genes and may thus provide precise phylogenetic information about closely related species. Moreover, disagreement between the results of 16S rRNA sequence and DNA-DNA hybridization studies has been described in detail by Stackebrandt and Goebel (19). An interesting example is the almost complete identity of the 16S rRNA genes derived from Bacillus anthracis and Bacillus cereus although the two species are well-differentiated on the basis of DNA-DNA hybridization data and phenotypic traits (9). Martinez-Murcia et al. (16) found a similar discrepancy in their study of the genus Aeromonas.

We tried to identify sequence stretches with diagnostic potential. The current investigation was based on direct sequence analysis of PCR-amplified $16 \mathrm{~S}$ rDNA. This approach produces a consensus 16S rDNA sequence for each strain studied, and it may be possible to detect sequence microheterogeneities (polymorphisms) due to nucleotide differences between $16 \mathrm{~S}$ rRNA genes which may be present in multiple copies in the bacterial genome. Our results have the potential for differentiating Acinetobacter spp. not only for reliable identification of an isolate as a member of the genus Acinetobacter, but also for placement of an isolate in the proper DNA group. This is possible because of unambiguous sequence differences in highly variable regions (22), especially in the areas characterized by helices 6 and 18 (22), corresponding to positions 
around positions 70 to 100 (Fig. 2) and 450 to 480 (Fig. 3) (IUB nomenclature for $E$. coli), respectively. The representative of each of the 21 DNA groups had a stretch of unique sequence motifs either in one region or in both regions. To our knowledge, this is the first time that the 21 DNA groups have been clearly differentiated. In our hands, when one reverse primer targeting positions 536 to 519 (IUB nomenclature for $E$. coli) was used, it was possible to read the complete stretch to the $5^{\prime}$ end of the PCR-amplified fragments encompassing both highly variable regions. Consequently, the use of this sequencing tool, coupled with automated fluorescent sequencing, should allow identification of unknown isolates as members of the genus Acinetobacter through the more conserved regions of partially analyzed $16 \mathrm{~S}$ rRNA genes and reliable and rapid DNA group differentiation based on the two highly variable areas characterized by helices 6 and 18 (22).

In conclusion, our data confirmed that the 21 Acinetobacter DNA groups form a coherent group of organisms. We also documented the intrageneric relationships of certain DNA groups, especially DNA groups 8 and 9. DNA groups 8 and 9 are indistinguishable phenotypically (1) and by most molecular methods, including DNA-DNA hybridization with the hydroxyapatite method (21), and we propose that they should be included in one species, $A$. Iwoffii. However, our delineation of intrageneric relationships revealed some disagreement with previous DNA-DNA hybridization data. Our findings also highlighted the potential of using direct sequencing of PCRamplified 16S rDNA as a rapid tool for identification and delineation of Acinetobacter strains. A final conclusion on this point cannot be drawn until more representatives of each DNA group are sequenced.

\section{ACKNOWLEDGMENTS}

We thank P. J. M. Bouvet, Institut Pasteur, for providing the reference strains for DNA groups BJ14 through BJ17 and Ingela Tjernberg, Malmö General Hospital, for providing the reference strains for DNA groups 12 and TU15.

\section{REFERENCES}

1. Bouvet, P. J. M., and P. A. D. Grimont. 1986. Taxonomy of the genus Acinetobacter with the recognition of Acinetobacter baumannii sp. nov., Acinetobacter haemolyticus sp. nov., Acinetobacter johnsonii sp. nov., and Acinetobacter junii sp. nov. and emended description of Acinetobacter calcoaceticus and Acinetobacter lwoffii. Int. J. Syst. Bacteriol. 36:228-240.

2. Bouvet, P. J. M., and S. Jeanjean. 1989. Delineation of new proteolytic genomic species in the genus Acinetobacter. Res. Microbiol. 140:291-299.

3. Brosius, J., J. L. Palmer, H. P. Kennedy, and H. F. Noller. 1978. Complete nucleotide sequence of a $16 \mathrm{~S}$ ribosomal RNA gene from Escherichia coli. Proc. Natl. Acad. Sci. USA 75:4801-4805.
4. Cilia, V., B. Lafay, and R. Christen. 1996. Sequence heterogeneities among 16S ribosomal RNA sequences and their effect on phylogenetic analyses at the species level. Mol. Biol. Evol. 13:451-461.

5. Emmert, D. B., P. J. Stoehr, G. Stoessner, and G. N. Cameron. 1994. The European Bioinformatics Institute (EBI databases). Nucleic Acids Res. 22: 3445-3449.

6. Enright, M. C., P. E. Carter, I. A. MacLean, and H. McKenzie. 1994. Phylogenetic relationships between some members of the genera Neisseria, Acinetobacter, Moraxella, and Kingella based on partial 16S ribosomal DNA sequence analysis. Int. J. Syst. Bacteriol, 44:387-391.

7. Felsenstein, J. 1985. Confidence limits on phylogenies: an approach using the bootstrap. Evolution 39:783-791.

8. Felsenstein, J. 1993. PHYLIP: phylogeny inference package (version 3.5c). University of Washington, Seattle.

9. Fox, G. E., J. D. Wisotzkey, and P. Jurtshuk, Jr. 1992. How close is close: 16S rRNA sequence identity may not be sufficient to guarantee species identity. Int. J. Syst. Bacteriol. 42:166-170.

10. Gerner-Smidt, P., I. Tjernberg, and J. Ursing. 1991. Reliability of phenotypic tests for identification of Acinetobacter species. J. Clin. Microbiol. 29:277-282.

11. Gerner-Smidt, P., and I. Tjernberg. 1993. Acinetobacter in Denmark. II Molecular studies of the Acinetobacter calcoaceticus-Acinetobacter baumannii complex. APMIS 101:826-832.

12. Ibrahim, A., B. M. Goebel, W. Liesack, M. Griffiths, and E. Stackebrandt. 1993. The phylogeny of the genus Yersinia based on 16S rDNA sequences. FEMS Microbiol. Lett. 114:173-178.

13. Jukes, T. H., and C. R. Cantor. 1969. Evolution of protein molecules, p. 21-132. In H. N. Munro (ed.), Mammalian protein metabolism, vol. 3. Academic Press, New York, N.Y.

14. Lane, D. J. 1991. 16S/23S rRNA sequencing, p. 115-175. In E. Stackebrandt and M. Goodfellow (ed.), Nucleic acid techniques in bacterial systematics. John Wiley \& Sons, New York, N.Y.

15. Maidak, B. L., N. Larsen, M. J. McCaughey, R. Overbeek, G. J. Olsen, K. Fogel, J. Blandy, and C. R. Woese. 1994. The Ribosomal Database Project. Nucleic Acids Res. 22:3485-3487.

16. Martinez-Murcia, A. J., S. Belloch, and M. D. Collins. 1992. Phylogenetic interrelationship of members of the genera Aeromonas and Plesiomonas as determined by $16 S$ ribosomal DNA sequence: lack of congruence with results of DNA-DNA hybridization. Int. J. Syst. Bacteriol, 42:412-421.

17. Nishimura, Y., T. Ino, and H. Hzuka. 1988. Acinetobacter radioresistens sp. nov. isolated from cotton and soil. Int. J. Syst. Bacteriol. 38:209-211.

18. Rainey, F. A., E. Lang, and E. Stackebrandt. 1994. The phylogenetic structure of the genus Acinetobacter. FEMS Microbiol. Lett. 124:349-354.

19. Stackebrandt, E., and B. M. Goebel. 1994. Taxonomic note: a place for DNA-DNA reassociation and 16S rRNA sequence analysis in the present species definition in bacteriology. Int. J. Syst. Bacteriol. 44:846-849.

20. Stackebrandt, E., W. Liesack, and D. Witt. 1992. Ribosomal RNA and ribosomal DNA sequence analysis. Gene 115:255-260.

21. Tjernberg, I., and J. Ursing. 1989. Clinical strains of Acinetobacter classified by DNA-DNA hybridization. APMIS 97:595-605.

22. Van de Peer, Y., S. Chapelle, and R. De Wachter. 1996. A quantitative map of nucleotide substitution rates in bacterial rRNA. Nucleic Acids Res. 24: 3381-3391.

23. Van de Peer, Y., S. Nicolaï, P. De Rijk, and R. De Wachter. 1996. Database on the structure of small ribosomal subunit RNA. Nucleic Acids Res. 24: 86-91.

24. Yamamoto, S., and S. Harayama. 1996. Phylogenetic analysis of Acinetobacter strains based on the nucleotide sequences of gyr $B$ genes and on the amino acid sequences of their products. Int. J. Syst. Bacteriol. 46:506-511. 